\title{
Numerical simulation of liquid round jet atomization
}

\author{
Dorrin Jarrahbashi, ${ }^{1,}{ }^{*}$ William A. Sirignano, ${ }^{2}$ Pavel P. Popov, ${ }^{3}$ and Fazle Hussain ${ }^{4}$ \\ ${ }^{1}$ Department of Mechanical Engineering, Georgia Institute of Technology, Atlanta, Georgia 30332, USA \\ ${ }^{2}$ Department of Mechanical and Aerospace Engineering, University of California, Irvine, \\ California 92697, USA \\ ${ }^{3}$ Coordinated Science Laboratory, University of Illinois at Urbana-Champaign, Urbana, Illinois 61801, USA \\ ${ }^{4}$ Department of Mechanical Engineering, Texas Tech University, Lubbock, Texas 79409, USA
}

(Received 28 June 2017; published 29 September 2017)

\begin{abstract}
This paper is associated with a poster winner of a 2016 APS/DFD Gallery of Fluid Motion Award for work presented at the DFD Gallery of Fluid Motion. The original poster is available from the Gallery of Fluid Motion, https://doi.org/10.1103/APS.DFD.2016. GFM.P0009
\end{abstract}

DOI: 10.1103/PhysRevFluids.2.090504

The breakup of liquid jets during injection (i.e., atomization) significantly affects the effectiveness of the application (e.g., combustion of liquid fuel, coating, or agricultural spraying). The droplet size and velocity distributions and spray shape resulting from the primary atomization process of liquid round jets are related to the cascade of small liquid structures that form during that process. The droplets break from these structures due to capillary action. Direct numerical simulation of the spray formation process for a round liquid jet segment with an outer, coaxial, high-density gas flow reveals holes, bridges, and ligaments on the conical wave crests at the liquid-gas interface. Temporal instabilities and liquid surface shape have been identified by solving three-dimensional incompressible Navier-Stokes equations on a Cartesian grid and using the level-set method for tracking the liquid-gas interface for wide ranges of the density ratio and Reynolds $(\mathrm{Re})$ and Weber (We) numbers [1]. Re and We have been defined based on the liquid density, liquid viscosity, liquid jet diameter, coaxial gas velocity, and liquid-gas surface tension.

Three physical domains of atomization are depicted in Fig. 1. At higher Re and We as in Fig. 1(a), four lobes form initially on the conical wave crests as the natural mode of instability (without forcing). Later, two holes appear off center on both sides of each lobe, and holes extend to the vicinity of the lobe rims and form very narrow bridges. Finally, the ligaments and then droplets develop following breakup of the bridges and ligaments, respectively. At higher gas densities, four lobes form on the conical crests throughout the entire Re range, as shown in Fig. 1(b). However, the lobes on the crests have lower curvature in the azimuthal direction and extend less in the gas-stream direction, causing holes on neighboring lobes to merge before the rims break to form ligaments. Two bridges per lobe, denoted as the middle and rim bridges, appear before breaking to form two ligaments and one center droplet per bridge. For lower gas density and lower Re or We as in Fig. 1(c), the well-ordered lobes are replaced by irregular, small-scale corrugations along the conical wave crest edge from which ligaments grow and extend radially outward. This behavior is similar to the experimental results of Marmottant and Villermaux [2] for a water jet with coaxial air flow at atmospheric and low-Re conditions. A more developed spray with a large number of ligaments, holes, and droplets with various length scales for the same conditions as in Fig. 1(c) at a later snapshot of liquid-gas interface is shown in Fig. 1(d). The thicknesses of liquid bridges and ligaments are significant in determining

\footnotetext{
*Corresponding author: dorrin.jarrahbashi@me.gatech.edu
}

Published by the American Physical Society under the terms of the Creative Commons Attribution 4.0 International license. Further distribution of this work must maintain attribution to the author(s) and the published article's title, journal citation, and DOI. 


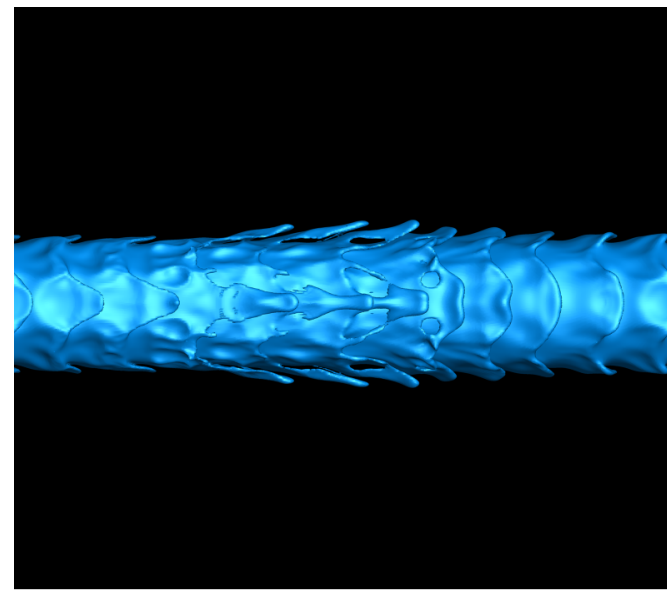

(a)

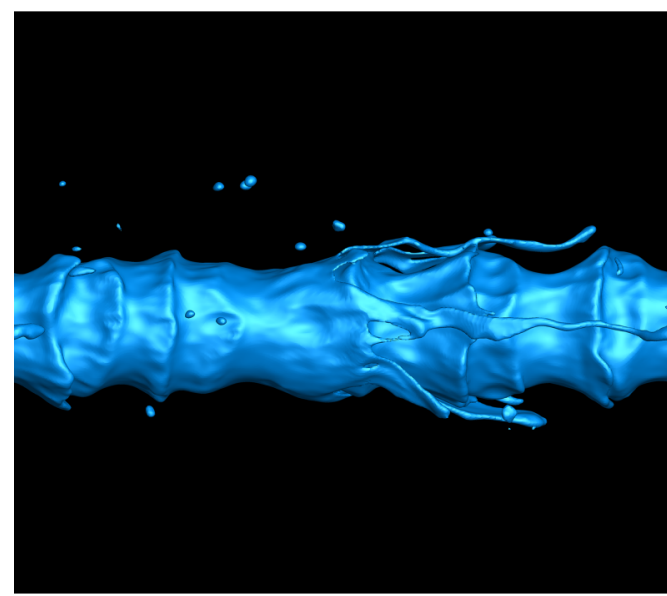

(c)

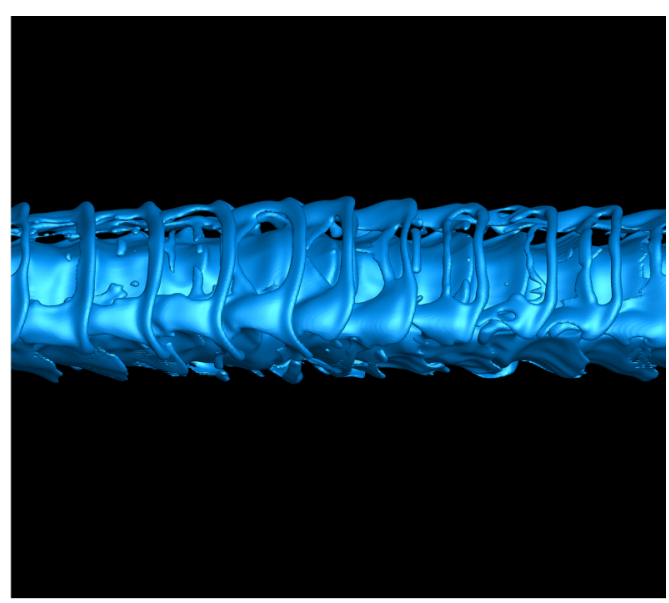

(b)

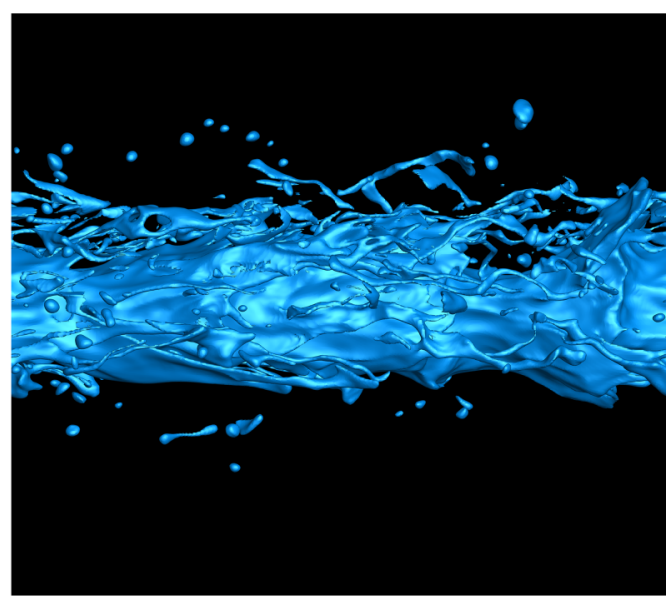

(d)

FIG. 1. Deformation of the liquid jet surface exposed to high-velocity coaxial gas flow demonstrating three different regimes of the primary breakup and spray formation from early development of lobes and holes to formation of the ligaments and droplets. (a) $\operatorname{Re}=1600, \mathrm{We}=230000, \rho_{g} / \rho_{l}=0.1$. (b) $\operatorname{Re}=1600$, $\mathrm{We}=230000, \rho_{g} / \rho_{l}=0.5$. (c) $\mathrm{Re}=320, \mathrm{We}=230000, \rho_{g} / \rho_{l}=0.05$. (d) Late-time spray development for the third regime of primary breakup of (c).

the early droplet sizes. With increasing gas density and increasing liquid viscosity, thicker ligaments and larger droplets occur in the ranges of low Re and of low gas density. At higher gas densities and Re values shown in Fig. 1, the spray extends radially outward more since the radially outward velocity component increases with gas density.

The hole formation process in Figs. 1(a) and 1(b) is correlated with the structure of hairpin and helical vortices on the lobes [3]. Postprocessing the results and projecting the vortex line at the liquid-gas interface indicated that the hairpin vortices first appeared in the braid region, i.e., between two adjacent Kelvin-Helmholtz cone crests, extended in the streamwise direction and wrapped around the hairpin vortices on the cone crest itself, in a fashion similar to the vortical mixing layer structures observed by Bernal and Roshko [4]. The mutual rotation of the vortex lines after overlapping of hairpin vortices pushes the top surface of the liquid sheet downward and the bottom sheet surface upward. This causes the liquid sheet to become thinner and puncture in that region to 


\section{NUMERICAL SIMULATION OF LIQUID ROUND JET . . .}

form holes at higher We, at higher gas-to-liquid density ratio, and in a wide Re range. The vorticity structures shown in Figs. 1(c) and 1(d) are not as regular as in the high-gas-density and high-Re cases shown in Figs. 1(a) and 1(b). Qualitatively similar results have been shown for the planar jet [5].

Access to the NICS Kraken supercomputer under Allocation TG-CTS130062 and to the UCI HPC cluster was very valuable in performing our high-resolution computations.

[1] D. Jarrahbashi and W. A. Sirignano, Vorticity dynamics for transient high-pressure liquid injection, Phys. Fluids 26, 101304 (2014).

[2] P. Marmottant and E. Villermaux, On spray formation, J. Fluid Mech. 498, 73 (2004).

[3] D. Jarrahbashi, W. A. Sirignano, P. P. Popov, and F. Hussain, Early spray development at high gas density: Hole, ligament and bridge formations, J. Fluid Mech. 792, 186 (2016).

[4] L. P. Bernal and A. Roshko, Streamwise vortex structure in plane mixing layers, J. Fluid Mech. 170, 499 (2006).

[5] A. Zandian, W. A. Sirignano, and F. Hussain, Planar liquid jet: Early deformation and atomization cascades, Phys. Fluids 29, 062109 (2017). 